\title{
On the generalized geometry origin of noncommutative gauge theory
}

\author{
Branislav Jurčo, ${ }^{a, b}$ Peter Schupp ${ }^{c}$ and Jan Vysokýc,d \\ ${ }^{a}$ Mathematical Institute, Faculty of Mathematics and Physics, Charles University, \\ Prague 186 75, Czech Republic \\ ${ }^{b}$ CERN, Theory Division, \\ CH-1211 Geneva 23, Switzerland \\ c Jacobs University Bremen, \\ 28759 Bremen, Germany \\ ${ }^{d}$ Czech Technical University in Prague, \\ Faculty of Nuclear Sciences and Physical Engineering, \\ Prague 115 19, Czech Republic \\ E-mail: jurco@karlin.mff.cuni.cz, p.schupp@jacobs-university.de, \\ vysokjan@fjfi.cvut.cz
}

ABSTRACT: We discuss noncommutative gauge theory from the generalized geometry point of view. We argue that the equivalence between the commutative and semiclassically noncommutative DBI actions is naturally encoded in the generalized geometry of D-branes.

KeYwords: D-branes, Non-Commutative Geometry, Differential and Algebraic Geometry, Sigma Models 


\section{Contents}

1 Introduction 1

2 Generalized geometry 3

2.1 Fiberwise metric, generalized metric 3

$\begin{array}{lll}2.2 & \text { Factorizations of generalized metric, open-closed relations } & 6\end{array}$

$\begin{array}{lll}2.3 & \text { Dorfman bracket, Dirac structures, D-branes } & 7\end{array}$

3 Gauge field as an orthogonal transformation of the generalized metric 8

4 Non-topological Poisson-sigma model and Polyakov action 10

$\begin{array}{llr}5 & \text { Seiberg-Witten map } & 11\end{array}$

6 Noncommutative gauge theory and DBI action 12

\section{Introduction}

Generalized geometry [1, 2] recently appeared to be a powerful mathematical tool for the description of various aspects of string and field theories. Here we mention only few instances of its relevance that are more or less directly related to the present paper. Topological and non-topological Poisson sigma models are known to be intimately related to a lot of interesting differential, in particular generalized, geometry. For instance, the topological Poisson sigma models are of interest for the integration of Poisson manifolds (and Lie algebroids) [3] and are at the heart of deformation quantization [4]. Field equations of (topological) Poisson sigma models can be interpreted as Lie algebroid morphisms [5] and as such can further be generalized in terms of generalized (complex) geometry [6, 7]. Poisson sigma models can be twisted by a 3 -form $H$-field [8] and also generalized to Dirac sigma models [7], where the graph defined by the corresponding (possibly twisted) Poisson structure is replaced by a more general Dirac structure. In turn, at least in some instances, D-branes can be related to Dirac structures [9, 10], or coisotropic submanifolds [11]. In [12], it has been observed that the current algebra of sigma models naturally involves structures of generalized geometry, such as the Dorfman bracket and Dirac structures. This was further developed in [13] and [14]. In [15], it was observed that in the first order (nontopological) Poisson sigma model characterized by a 2 -form $B$ and a bivector $\theta$, a more general form of world-sheet currents appears. Their algebra has been shown to close under a more general bracket, the so called Roytenberg bracket [16]. In [17], it has been shown that the structure constants of the Roytenberg bracket appear if one lifts the topological 
part of first order Poisson sigma characterized by a 2 -form $B$ and a bivector $\theta$ to a threedimensional WZW term. It this respect, generalized geometry is relevant for discussions of non-geometric backgrounds.

Noncommutativity of open strings, more precisely of their endpoints, in the presence of a $B$-field was recognized in $[18,19]$ and $[20]$. A thorough discussion of noncommutativity in string theory followed in the famous article of Seiberg and Witten [21], where, among other things, also the equivalence of commutative and noncommutative gauge theories was discussed via a field redefinition known under the name Seiberg-Witten map. In particular, it was argued that the higher derivative terms in the noncommutative version of the Dirac-Born-Infeld (DBI) action can be viewed as corrections to the usual DBI action, the effective D-brane action. For reviews on noncommutativity in string theory we refer, e.g., to $[22,23]$. Let us also note that the (semiclassical) noncommutativity of D-branes can be seen as the (semiclassical) noncommutativity of the string endpoints in the open topological Poisson sigma model [3], which fits naturally to their role in both the integration as well as deformation quantization of Poisson structures.

The purpose of the present paper is to unravel the generalized geometry origin of noncommutative gauge theory. We will mainly focus on the equivalence between the commutative and semiclassically noncommutative DBI actions (and closely related issues) and argue that the necessity of such an equivalence can be seen and naturally interpreted within generalized geometry. In the discussion, non-topological Poisson sigma models play a role. Roughly speaking, we intend to convince the reader that the equivalence of commutative and semiclassically noncommutative DBI actions is encoded in two different ways of expressing a generalized metric on a D-brane.

Before going into a more detailed description of the individual sections, let us note that almost everything in this paper is presented in a form suitable for a direct generalization to Nambu-Poisson structures and M-theory membranes, cf. [24, 25]. We will discuss this in detail in a forthcoming paper.

The paper is organized as follows.

In the second section, we review basic definitions of generalized geometry. We emphasize the behavior of a generalized metric under orthogonal transformations of $T M \oplus T^{*} M$. This allows us to recover the formulas relating, via a bivector $\theta$, the closed background fields $g, B$ and the open string backgrounds $G$ and $\Phi$. It comes as a relation between two generalized metrics, which are connected by the action of a certain orthogonal transformation induced by the bivector $\theta$. Finally, we recall the definition of the Dorfman bracket, Dirac structures and their relation to D-branes. In the latter we follow the proposal of [10], where D-branes correspond to leaves of foliations defined by Dirac structures.

In the third section, we observe that adding the gauge field $F$ on D-brane volume corresponds to an action of an orthogonal transformation on the natural generalized metric on the D-brane, the pullback of the generalized space-time metric defined by the closed backgrounds $g$ and $B$. The natural question is whether the so obtained generalized metric can again be rewritten in the open string variables (with some gauge field $F^{\prime}$ and a possibly modified bivector $\theta^{\prime}$ ). The positive answer is given by two different factorizations of an orthogonal transformations defined by a bivector and a 2 -form, in our case $\theta$ and $F$. As 
a consequence, we find a generalization of open-closed relations of Seiberg and Witten, which includes the field strengths $F$ and $F^{\prime}$, the latter one closely related to the nocommutative gauge field strength. This equality, crucial for our discussion of DBI actions, also hints towards the appearance of the semiclassical Seiberg-Witten map, once one recalls its interpretation as the local coordinate change between the two (Poisson) bivectors $\theta$ and $\theta^{\prime}$.

In the fourth section, we use the above mentioned relation between open and closed variables (including gauge fields) to show that non-topological Poisson-sigma model, its Hamiltonian and the corresponding Polyakov action are manifestly invariant under the open-closed field redefinitions as they geometrically correspond to the same generalized metric.

In the fifth section, we briefly recall the interpretation of the semiclassical SeibergWitten map as a local diffeomorphism on the D-brane world volume relating the noncommutativity parameters (Poisson bivectors) $\theta$ and $\theta^{\prime}$. This interpretation is the most relevant one for our discussion in the final section. When considering D-branes which are symplectic leaves of $\theta$, the Seiberg-Witten map is naturally interpreted in terms of the corresponding Dirac structure.

In the final section, we discuss the equivalence of commutative and semiclassically noncommutative DBI action of a D-brane. We show that this equivalence is a direct consequence of the (gauge field dependent) open-closed relations combined with a SeibergWitten map. The discussion here is not completely new. However, what we believe is new and interesting is the clear generalized geometry origin of its main ingredients as developed in previous sections. Everything works very naturally for a D-brane which is a symplectic leaf of the Poisson structure, describing the noncommutativity.

We believe that analogous results hold also for more general $D$-branes, i.e. those which are related to more general Dirac structures than the ones defined by graphs of Poisson tensors. For such D-branes, Dirac sigma models of [7] should replace the Poisson sigma models.

\section{Generalized geometry}

\subsection{Fiberwise metric, generalized metric}

In this section we recall some basic facts regarding generalized geometry, see, e.g., [2, 26]. Although most of the involved objects can be defined in a more general framework, we focus on a particular choice of vector bundle. Namely, let $M$ be a smooth manifold and $E=T M \oplus T^{*} M$. A fiberwise metric $(\cdot, \cdot)$ on $E$ is a $C^{\infty}(M)$-bilinear map $(\cdot, \cdot): \Gamma(E) \times$ $\Gamma(E) \rightarrow C^{\infty}(M)$, such that for each $p \in M,(\cdot, \cdot)_{p}: E_{p} \times E_{p} \rightarrow \mathbb{R}$ is a symmetric nondegenerate bilinear form. There exists a canonical fiberwise metric $\langle\cdot, \cdot\rangle$ on $E$, defined as

$$
\langle V+\xi, W+\eta\rangle=i_{V}(\eta)+i_{W}(\xi),
$$

for every $(V+\xi),(W+\eta) \in \Gamma(E)$. This fiberwise metric has signature $(n, n)$, where $n$ is a dimension of $M$. Hence, we denote by $O(n, n)$ the set of vector bundle automorphisms preserving this fiberwise metric. That is

$$
O(n, n)=\left\{O \in \Gamma(\operatorname{Aut}(E)) \mid\left(\forall e_{1}, e_{2} \in \Gamma(E)\right)\left(\left\langle O e_{1}, O e_{2}\right\rangle=\left\langle e_{1}, e_{2}\right\rangle\right)\right\} .
$$


There are three important examples of $O(n, n)$ transformations, which we will use in the sequel. Let $B \in \Omega^{2}(M)$ be a 2 -form on $M$. In this paper we will always denote the induced vector bundle morphism from $T M$ to $T^{*} M$ by the same letter, i.e., we define

$$
B(V)=-i_{V} B=B(\cdot, V)
$$

for all $V \in \mathfrak{X}(M)$. Correspondingly, the map $e^{B}$ is given as

$$
e^{B}(V+\xi)=V+\xi+B(V) .
$$

In the block matrix form

$$
e^{B}\left(\begin{array}{l}
V \\
\xi
\end{array}\right)=\left(\begin{array}{ll}
1 & 0 \\
B & 1
\end{array}\right)\left(\begin{array}{l}
V \\
\xi
\end{array}\right),
$$

for all $(V+\xi) \in \Gamma(E)$. Similarly, let $\theta \in \Lambda^{2} \mathfrak{X}(M)$ be a bivector. The induced vector bundle morphism is again denoted by the same letter, that is

$$
\theta(\xi):=-i_{\xi} \theta=\theta(\cdot, \xi)
$$

for all $\xi \in \Omega^{1}(M)$. Correspondingly, we have $e^{\theta}$

$$
e^{\theta}(V+\xi)=V+\xi+\theta(\xi)
$$

In the block matrix form

$$
e^{\theta}\left(\begin{array}{l}
V \\
\xi
\end{array}\right)=\left(\begin{array}{ll}
1 & \theta \\
0 & 1
\end{array}\right)\left(\begin{array}{l}
V \\
\xi
\end{array}\right),
$$

for all $(V+\xi) \in \Gamma(E)$. Finally, let $N: T M \rightarrow T M$ be any invertible smooth vector bundle morphism over identity. We define the map $O_{N}$ as

$$
O_{N}(V+\xi):=N(V)+N^{-T}(\xi)
$$

where $N^{-T}: T^{*} M \rightarrow T^{*} M$ denotes the map transpose to $N^{-1}$. In the block matrix form

$$
O_{N}\left(\begin{array}{l}
V \\
\xi
\end{array}\right)=\left(\begin{array}{cc}
N & 0 \\
0 & N^{-T}
\end{array}\right)\left(\begin{array}{l}
V \\
\xi
\end{array}\right) .
$$

Any $O(n, n)$ transformation with the invertible upper-left block can be uniquely decomposed as a product of the form

$$
e^{-B} O_{N} e^{-\theta}
$$

More explicitly, for $\left(\begin{array}{cc}A_{11} & A_{12} \\ A_{21} & A_{22}\end{array}\right)$ in $O(n, n)$, i.e., $A_{21}^{T} A_{11}+A_{11}^{T} A_{21}=0, A_{12}^{T} A_{22}+A_{22}^{T} A_{12}=0$ and $A_{21}^{T} A_{12}+A_{11}^{T} A_{22}=1$, we find $N=A_{11}, \theta=-A_{11}^{-1} A_{12}$ and $B=-A_{21} A_{11}^{-1}$.

Let now $\tau: \Gamma(E) \rightarrow \Gamma(E)$ be a $C^{\infty}(M)$-linear map of sections, such that $\tau^{2}=1$. For $e_{1}, e_{2} \in \Gamma(E)$, we put

$$
\left(e_{1}, e_{2}\right)_{\tau}:=\left\langle\tau\left(e_{1}\right), e_{2}\right\rangle .
$$


If such $(., .)_{\tau}$ defines a positive definite fiberwise metric, we refer to it as a generalized metric on $E$. From now on, we will always assume that this is the case. Since $(\cdot, \cdot)_{\tau}$ is symmetric, $\tau$ is a symmetric map, that is,

$$
\left\langle\tau\left(e_{1}\right), e_{2}\right\rangle=\left\langle e_{1}, \tau\left(e_{2}\right)\right\rangle,
$$

for all $e_{1}, e_{2} \in \Gamma(E)$. Also, because $\tau^{2}=1$, it is orthogonal and thus $\tau \in O(n, n)$. Moreover, from $\tau^{2}=1$, we get two eigenbundles $V_{+}$and $V_{-}$, corresponding to +1 and -1 eigenvalues of $\tau$, respectively. Using the fact that $(\cdot, \cdot)_{\tau}$ is positive definite, we get that $\langle\cdot, \cdot\rangle$ is positive definite on $\Gamma\left(V_{+}\right)$and negative definite on $\Gamma\left(V_{-}\right)$. Finally, we can observe that $V_{+}^{\perp}=V_{-}$ with respect to $\langle\cdot, \cdot\rangle$ and vice versa, and using the knowledge of the signature of $\langle\cdot, \cdot\rangle$, we get the direct sum decomposition

$$
E=V_{+} \oplus V_{-} .
$$

Conversely, for any subbundle $V$ of $E$ of rank $n$, on which $\langle\cdot, \cdot\rangle$ is positive definite, set $\left.\tau\right|_{V}:=+1$ and $\left.\tau\right|_{V^{\perp}}=-1$ to get a generalized metric on $E$.

From positive definiteness on $V_{+}$, we have $V_{+} \cap T M=0$ and $V_{+} \cap T^{*} M=0$, and the same for $V_{-}$. This means that $V_{+}$and $V_{-}$can be viewed as graphs of invertible smooth vector bundle morphisms:

$$
\begin{aligned}
& V_{+}=\{V+A(V) \mid V \in T M\} \equiv\left\{A^{-1}(\xi)+\xi \mid \xi \in T^{*} M\right\}, \\
& V_{-}=\left\{V+A^{\prime}(V) \mid V \in T M\right\} \equiv\left\{A^{\prime-1}(\xi)+\xi \mid \xi \in T^{*} M\right\},
\end{aligned}
$$

where $A, A^{\prime}: T M \rightarrow T^{*} M$, respectively. We can view $A$ as covariant 2-tensor field on $M$, and write uniquely $A=g+B$, where $g$ is a symmetric part of $A$ and $B$ a skew-symmetric part of $A$. From the positive definiteness of $V_{+}$we get that $g$ is a Riemannian metric on $M$, whereas $B$ can be an arbitrary 2-form on $M$. Using the orthogonality of $V_{+}$and $V_{-}$, we see that $A^{\prime}=-g+B$. From this equivalent formulation, i.e. using $g$ and $B$, we can uniquely reconstruct $\tau$. This will give

$$
\tau(V+\xi)=\left(g-B g^{-1} B\right)(V)-g^{-1} B(V)+B g^{-1}(\xi)+g^{-1}(\xi),
$$

for all $(V+\xi) \in \Gamma(E)$. In the block matrix form,

$$
\tau\left(\begin{array}{l}
V \\
\xi
\end{array}\right)=\left(\begin{array}{cc}
-g^{-1} B & g^{-1} \\
g-B g^{-1} B & B g^{-1}
\end{array}\right)\left(\begin{array}{l}
V \\
\xi
\end{array}\right) .
$$

The corresponding fiberwise metric $(\cdot, \cdot)_{\tau}$ can then be written in the block matrix form

$$
(V+\xi, W+\eta)_{\tau}=\left(\begin{array}{c}
V \\
\xi
\end{array}\right)^{T}\left(\begin{array}{cc}
g-B g^{-1} B & B g^{-1} \\
-g^{-1} B & g^{-1}
\end{array}\right)\left(\begin{array}{c}
W \\
\eta
\end{array}\right)
$$

The important observation is that the block matrix in formula (2.19) can be written as a product of simpler matrices. Namely,

$$
\left(\begin{array}{cc}
g-B g^{-1} B & B g^{-1} \\
-g^{-1} B & g^{-1}
\end{array}\right)=\left(\begin{array}{ll}
1 & B \\
0 & 1
\end{array}\right)\left(\begin{array}{cc}
g & 0 \\
0 & g^{-1}
\end{array}\right)\left(\begin{array}{cc}
1 & 0 \\
-B & 1
\end{array}\right) .
$$


Note the important fact that the 2-form $B$ does not have to be closed, and this will remain true throughout the whole paper. Nevertheless, we assume that $B$ is globally defined, i.e. $H=d B$ globally. ${ }^{1}$ We thus consider only the models with trivial $H$-flux. The case of the non-trivial $H$-flux will be discussed elsewhere.

There exists a natural action of the group $O(n, n)$ on the space of generalized metrics. For each $O \in O(n, n)$ and given $\tau$ define $\tau^{\prime}=O^{-1} \tau O$. Clearly $\tau^{\prime 2}=1$ and

$$
\left\langle\tau^{\prime}\left(e_{1}\right), e_{2}\right\rangle=\left\langle\tau\left(O\left(e_{1}\right)\right), O\left(e_{2}\right)\right\rangle=\left(O\left(e_{1}\right), O\left(e_{2}\right)\right)_{\tau} .
$$

Hence $(\cdot, \cdot)_{\tau^{\prime}}$ is again a generalized metric. We may use the notation $(\cdot, \cdot)_{\tau^{\prime}}=O(\cdot, \cdot)_{\tau}$.

\subsection{Factorizations of generalized metric, open-closed relations}

Let us start with a (different) generalized metric $\mathbf{H}$, described by a Riemannian metric $G$ and a 2 -form $\Phi$. Hence

$$
\mathbf{H}=\left(\begin{array}{ll}
1 & \Phi \\
0 & 1
\end{array}\right)\left(\begin{array}{cc}
G & 0 \\
0 & G^{-1}
\end{array}\right)\left(\begin{array}{cc}
1 & 0 \\
-\Phi & 0
\end{array}\right) .
$$

Let $\theta$ be a 2-vector field on $M$. The action of the $O(n, n)$ map $e^{-\theta}$ on the generalized metric $\mathbf{H}$ gives us a new generalized metric $\mathbf{G}$, which has the form

$$
\mathbf{G}=\left(\begin{array}{ll}
1 & 0 \\
\theta & 1
\end{array}\right)\left(\begin{array}{ll}
1 & \Phi \\
0 & 1
\end{array}\right)\left(\begin{array}{cc}
G & 0 \\
0 & G^{-1}
\end{array}\right)\left(\begin{array}{cc}
1 & 0 \\
-\Phi & 1
\end{array}\right)\left(\begin{array}{cc}
1 & -\theta \\
0 & 1
\end{array}\right)
$$

By the previous discussion, there exists a unique Riemannian metric $g$ and a 2 -form $B$, such that

$$
\mathbf{G}=\left(\begin{array}{ll}
1 & B \\
0 & 1
\end{array}\right)\left(\begin{array}{cc}
g & 0 \\
0 & g^{-1}
\end{array}\right)\left(\begin{array}{cc}
1 & 0 \\
-B & 1
\end{array}\right)
$$

Comparing the two expressions (2.22) and (2.23) of $\mathbf{G}$, we get the matrix equations

$$
\begin{gathered}
g-B g^{-1} B=G-\Phi G^{-1} \Phi, \\
B g^{-1}=\Phi G^{-1}-\left(G-\Phi G^{-1} \Phi\right) \theta,
\end{gathered}
$$

which can be uniquely solved for $G$ and $\Phi$. Since $e^{-\theta}$ is invertible, we can proceed the other way around as well. We also know how the corresponding endomorphism $\tau_{\mathbf{H}}$ is changed by $e^{-\theta}$. Namely, we have

$$
\tau_{\mathbf{G}}=e^{\theta} \tau_{\mathbf{H}} e^{-\theta} .
$$

From that, we can easily find the relation between +1 eigenbundles:

$$
V_{+}^{\mathbf{G}}=e^{\theta} V_{+}^{\mathbf{H}} .
$$

Since

$$
V_{+}^{\mathbf{G}}=\left\{\xi+(g+B)^{-1}(\xi) \mid \xi \in T^{*} M\right\}
$$

\footnotetext{
${ }^{1}$ More precisely, we assume that the corresponding integral cohomology class $[H]$ is trivial.
} 
and

$$
V_{+}^{\mathbf{H}}=\left\{\xi+(G+\Phi)^{-1}(\xi) \mid \xi \in T^{*} M\right\},
$$

we get using the above formula that

$$
(g+B)^{-1}=\theta+(G+\Phi)^{-1} .
$$

Formulae (2.24) and (2.25) are the symmetric and antisymmetric parts of (2.28). If $\theta$ is Poisson, (2.28) is the Seiberg-Witten formula ${ }^{2}$ relating closed and open string backgrounds in the presence of a noncommutative structure represented by $\theta$. In particular, for given $g$, $B$ and $\theta$, we can find a unique $G$ and $\Phi$, and conversely, for given $G, \Phi$ and $\theta$, there exists a unique pair $g$ and $B$.

For $\Phi=0$ the open-closed relations can be given a slightly more geometric interpretation [10]. Consider the inverse $\mathbf{G}^{-1}$ of the generalized metric $\mathbf{G}$. If we exchange the tangent and cotangent bundles $T M$ and $T^{*} M$, respectively, $\mathbf{G}^{-1}$ has the same properties as $\mathbf{G}$. Obviously, $\mathbf{G}^{-1}$ and $\mathbf{G}$ have identical graphs as well as \pm 1-eigenbundles. The open-closed relations, for $\Phi=0$, is a simple consequence of that.

\subsection{Dorfman bracket, Dirac structures, D-branes}

Here we briefly recall some relevant facts concerning the Dorfman bracket and Dirac structures, see, e.g., $[2,26,28]$. Our vector bundle $E=T M \oplus T^{*} M$ can be equipped with a structure of a Courant algebroid. The corresponding Courant bracket is the antisymmetrization of the Dorfman bracket:

$$
[V+\xi, W+\eta]_{D}=[V, W]+\mathcal{L}_{V}(\eta)-i_{W}(d \xi)
$$

for all $(V+\xi) \in \Gamma(E)$. The corresponding pairing is the canonical fiberwise metric (2.1).

A Dirac structure is a (smooth) subbundle $L$ of $E$, which is maximally isotropic with respect to $\langle\cdot, \cdot\rangle$ and involutive under the Dorfman bracket $(2.29)$.

Let $\theta$ be a rank-2 contravariant tensor field on $M$. As before, define a vector bundle morphism $\theta: T^{*} M \rightarrow T M$ by $\theta(\xi)=\theta(\cdot, \xi)$. Define a subbundle $G_{\theta}$ of $E$ as its graph, that is

$$
G_{\theta}=\left\{\xi+\theta(\xi) \mid \xi \in T^{*} M\right\} .
$$

It is known that $G_{\theta}$ is a Dirac structure with respect to the Dorfman bracket, if and only if $\theta$ is a Poisson bivector. Similarly, let $B$ be any rank-2 covariant tensor field on $M$. Define $B(V)=B(V, \cdot)$ and its graph $G_{B}$ as

$$
G_{B}=\{V+B(V) \mid V \in T M\} .
$$

Again, one can show that $G_{B}$ is a Dirac structure, if and only if $B$ is a closed 2-form on $M$.

Furthermore, for any closed $B \in \Omega^{2}(M)$, one has

$$
e^{B}[V+\xi, W+\eta]_{D}=\left[e^{B}(V+\xi), e^{B}(W+\eta)\right]_{D},
$$

\footnotetext{
${ }^{2}$ For an earlier appearance of this type of formulae in the context of duality rotations see [27].
} 
and

$$
\left\langle e^{B}(V+\xi), e^{B}(W+\eta)\right\rangle=\langle V+\xi, W+\eta\rangle,
$$

for all $(V+\xi),(W+\eta) \in \Gamma(E)$. In the other words, $e^{B}$ is an automorphism of the corresponding Courant algebroid. Note that (2.32) is no longer true for $e^{\theta}$, where $\theta \in$ $\Lambda^{2} \mathfrak{X}(M)$, but $(2.33)$ holds.

Generally, a Dirac structure $L$ provides a singular foliation of $M$ by presympletic leaves, which is generated by its image $\rho(L)$ of the Dirac structure under the anchor map. We refer to [10] for arguments in favor of the identification "D-branes $\sim$ leaves of foliations defined by Dirac structures". In the case we will consider later, $L$ will be given as a graph of a Poisson tensor $\theta$ and the corresponding foliation of $M$ will be the foliation generated by Hamiltonian vector fields, i.e., by symplectic leaves of $\theta$. Hence, in this case we will identify the symplectic leaves and D-branes.

\section{Gauge field as an orthogonal transformation of the generalized metric}

Let us start with a given Riemannian metric $g$ and 2-form $B$. Further, let $F$ be a 2-form (at this point an arbitrary one ${ }^{3}$. The gauge transformation defines new 2-form $B^{\prime}=B+F$. To the pair $(g, B)$ corresponds the generalized metric $\mathbf{G}$, see (2.23). The generalized metric $\mathbf{G}^{\prime}$ corresponding to the pair $(g, B+F)$ has the following block matrix form:

$$
\mathbf{G}^{\prime}=\left(\begin{array}{ll}
1 & F \\
0 & 1
\end{array}\right)\left(\begin{array}{ll}
1 & B \\
0 & 1
\end{array}\right)\left(\begin{array}{cc}
g & 0 \\
0 & g^{-1}
\end{array}\right)\left(\begin{array}{cc}
1 & 0 \\
-B & 1
\end{array}\right)\left(\begin{array}{cc}
1 & 0 \\
-F & 1
\end{array}\right)
$$

that is, $\mathbf{G}^{\prime}$ is related to $\mathbf{G}$ by the $O(n, n)$ transform $e^{-F}$. As shown before, we can always get $\mathbf{G}$ by action of $O(n, n)$ transformation $e^{-\theta}$ on the generalized metric $\mathbf{H}$, where $\mathbf{H}$ is described by fields $G$ and $\Phi$, see (2.21).

One may ask, if there is a bivector $\theta^{\prime}$ on $M$, such that we get $\mathbf{G}^{\prime}$ by the action of $e^{-\theta^{\prime}}$ on the generalized metric $\mathbf{H}^{\prime}$, which is described by the same $G$ as $\mathbf{H}$, but by gauged 2-form $\Phi^{\prime}=\Phi+F^{\prime}$ for some gauge field $F^{\prime}$. This can be achieved under some assumptions, however, only up to a certain additional $O(n, n)$ action. In particular, there exists a vector bundle morphism $N: T M \rightarrow T M$, such that

$$
\mathbf{G}^{\prime}=\left(\begin{array}{ll}
1 & 0 \\
\theta^{\prime} & 1
\end{array}\right)\left(\begin{array}{cc}
N^{T} & 0 \\
0 & N^{-1}
\end{array}\right) \mathbf{H}^{\prime}\left(\begin{array}{cc}
N & 0 \\
0 & N^{-T}
\end{array}\right)\left(\begin{array}{cc}
1 & -\theta^{\prime} \\
0 & 1
\end{array}\right),
$$

where

$$
\mathbf{H}^{\prime}=\left(\begin{array}{ll}
1 & \Phi^{\prime} \\
0 & 1
\end{array}\right)\left(\begin{array}{cc}
G & 0 \\
0 & G^{-1}
\end{array}\right)\left(\begin{array}{cc}
1 & 0 \\
-\Phi^{\prime} & 1
\end{array}\right) .
$$

Indeed, examine the block matrix decomposition:

$$
\mathbf{G}^{\prime}=\left(\begin{array}{ll}
1 & F \\
0 & 1
\end{array}\right)\left(\begin{array}{ll}
1 & 0 \\
\theta & 1
\end{array}\right)\left(\begin{array}{ll}
1 & \Phi \\
0 & 1
\end{array}\right)\left(\begin{array}{cc}
G & 0 \\
0 & G^{-1}
\end{array}\right)\left(\begin{array}{cc}
1 & 0 \\
-\Phi & 1
\end{array}\right)\left(\begin{array}{cc}
1 & -\theta \\
0 & 1
\end{array}\right)\left(\begin{array}{cc}
1 & 0 \\
-F & 1
\end{array}\right) .
$$

\footnotetext{
${ }^{3}$ Later, when discussing DBI action, $F$ will be closed and defined only on a submanifold of $M$ supporting a D-brane. In which case, all expression involving $F$ will make sense only when considered on the D-brane.
} 
It suffices to consider the three rightmost matrices in the above expression. Since we want to modify $\Phi$ to $\Phi+F^{\prime}$, we may proceed by inserting $1=e^{-F^{\prime}} e^{F^{\prime}}$ :

$$
\left(\begin{array}{cc}
1 & 0 \\
-\Phi & 1
\end{array}\right)\left(\begin{array}{cc}
1 & -\theta \\
0 & 1
\end{array}\right)\left(\begin{array}{cc}
1 & 0 \\
-F & 1
\end{array}\right)=\left(\begin{array}{cc}
1 & 0 \\
-\left(\Phi+F^{\prime}\right) & 1
\end{array}\right)\left(\begin{array}{cc}
1 & 0 \\
F^{\prime} & 1
\end{array}\right)\left(\begin{array}{cc}
1 & -\theta \\
0 & 1
\end{array}\right)\left(\begin{array}{cc}
1 & 0 \\
-F & 1
\end{array}\right) \text {. }
$$

Now it is enough to note that the product of the last three matrices, can be uniquely decomposed into a product of a diagonal and an upper triangular block matrix - of course, only if we assume that $(1+\theta F)$ is invertible. For this, use the decomposition of $e^{-\theta} e^{-F} \in O(n, n)$ according to $(2.11)$ as

$$
e^{-\theta} e^{-F}=e^{-F^{\prime}} O_{N} e^{-\theta^{\prime}}
$$

with $F^{\prime} \in \Omega^{2}(M), \theta^{\prime} \in \Lambda^{2} \mathfrak{X}(M)$ and $N \in \Gamma(\operatorname{Aut}(T M))$. What we find are the following expression for $\theta^{\prime}, F^{\prime}$ and $N$ :

$$
\begin{aligned}
\theta^{\prime} & =(1+\theta F)^{-1} \theta=\theta(1+F \theta)^{-1}, \\
F^{\prime} & =F(1+\theta F)^{-1}=(1+F \theta)^{-1} F, \\
N & =1+\theta F .
\end{aligned}
$$

Comparing (3.1) and (3.2), we get the equalities

$$
g-(B+F) g^{-1}(B+F)=N^{T}\left(G-\left(\Phi+F^{\prime}\right) G^{-1}\left(\Phi+F^{\prime}\right)\right) N
$$

and

$$
(B+F) g^{-1}=N^{T}\left(\Phi+F^{\prime}\right) G^{-1} N^{-T}-N^{T}\left(G-\left(\Phi+F^{\prime}\right) G^{-1}\left(\Phi+F^{\prime}\right)\right) N \theta^{\prime} .
$$

Taking the determinant of (3.7), we find that

$$
\operatorname{det}\left(g-(B+F) g^{-1}(B+F)\right)=\operatorname{det}(N)^{2} \cdot \operatorname{det}\left(G-\left(\Phi+F^{\prime}\right) G^{-1}\left(\Phi+F^{\prime}\right)\right) .
$$

This equality will play the central role when later discussing the DBI action.

Furthermore, following the same type of arguments leading to (2.28) we see that the equations (3.7) and (3.8) can equivalently be written as

$$
(g+B+F)^{-1}=\theta^{\prime}+\left(N^{T}\left(G+\Phi+F^{\prime}\right) N\right)^{-1} .
$$

Finally, let us examine the objects $F^{\prime}$ and $\theta^{\prime}$ using the tools described in subsection 2.3. We will concentrate on the case important for the discussion of the DBI action and noncommutative gauge theory. Therefore, in the rest of this section, we assume that $\theta$ is Poisson and $F$ is closed. $\theta^{\prime}$ is a bivector on $M$. For the graphs of $\theta$ and $\theta^{\prime}$ we have

$$
e^{F} G_{\theta}=G_{\theta^{\prime}}
$$

Since $e^{F}$ is an automorphism of Dorfman bracket, $G_{\theta^{\prime}}$ has to be again a Dirac structure of $E$. Hence, $\theta^{\prime}$ is a Poisson bivector. Similarly, one can see that

$$
e^{\theta} G_{F}=G_{F^{\prime}} .
$$


This is no more an automorphism of Dorfman bracket but it preserves the (maximal) isotropy property of $G_{F}$. Hence $G_{F^{\prime}}$ is an isotropic subbundle of $E$ and $F^{\prime}$ is therefore a 2-form on $M$. Let us also note, that $F^{\prime}$ doesn't need to be closed. The last remark: In case that $(1+\theta F)$ is not invertible, $e^{F} G_{\theta}$ still makes perfect sense as a Dirac structure. Similarly, $e^{\theta} G_{F}$ will still define an almost Dirac structure.

\section{Non-topological Poisson-sigma model and Polyakov action}

In this section we review the non-topological Poisson-sigma model from the generalized geometry point of view developed in the previous sections.

Let us consider a 2-dimensional world-sheet $\Sigma$ with a set of local coordinates $\left(\sigma^{0}, \sigma^{1}\right)$. We assume that $\sigma^{\mu}$ are Cartesian coordinates for a Lorentzian metric $h$ with signature $(-,+)$ on $\Sigma$. Furthermore, we consider an $n$-dimensional target manifold $M$, equipped with a metric $G, 2$-vector $\theta$ and a 2 -form $\Phi$. We can assume $\Sigma$ with a non-empty boundary $\partial \Sigma$. On $M$ assume an abelian gauge field $A$ coupling to the boundary (and extending to $\Sigma$, the field strength being $F=d A$ ). We also choose some local coordinates $\left(y^{1}, \ldots, y^{n}\right)$ on $M$. Lower case Latin characters will always correspond to these coordinates. For the components of the smooth map $X: \Sigma \rightarrow M$ we will use the following notation: $X^{i}=y^{i}(X)$. In this section it will be convenient to introduce the following notation: We put $\bar{G}:=N^{T} G N, \bar{\Phi}:=N^{T} \Phi N$ and $\bar{F}^{\prime}:=N^{T} F^{\prime} N$ and introduce auxiliary fields $\eta_{i}$ and $\widetilde{\eta}_{j}$, which transform under change of local coordinates on $M$ according to their index structure. We combine them in a $2 n$-dimensional column vector $\Psi^{T}:=(\eta, \tilde{\eta})$. We also introduce another $2 n$-dimensional column vector $V^{T}:=\left(\partial_{0} X, \partial_{1} X\right)$. Finally, we define a $2 n \times 2 n$ matrix $^{4}$

$$
\overline{\mathbf{G}}=\left(\begin{array}{cc}
-\bar{G} & -\bar{\Phi}-\bar{F}^{\prime} \\
\bar{\Phi}+\bar{F}^{\prime} & \bar{G}
\end{array}\right)^{-1}+\left(\begin{array}{cc}
0 & \theta^{\prime} \\
-\theta^{\prime} & 0
\end{array}\right)
$$

Our (non-topological) Poisson-sigma model action is

$$
S[\eta, \widetilde{\eta}, X]:=\int d^{2} \sigma \frac{1}{2} \Psi^{T} \overline{\mathbf{G}} \Psi+\Psi^{T} V
$$

Using relations (3.7), (3.8), the action (4.2) can equivalently be written as

$$
S[\eta, \widetilde{\eta}, X]:=\int d^{2} \sigma \frac{1}{2} \Psi^{T} \tilde{\mathbf{G}} \Psi+\Psi^{T} V,
$$

where

$$
\tilde{\mathbf{G}}=\left(\begin{array}{cc}
-g & -B-F \\
B+F & g
\end{array}\right)^{-1}
$$

with $g, B$ and $F$ being related to $G, \Phi$ and $F^{\prime}$ by (3.7), (3.8) and (3.5). Integrating out the auxiliary fields $\eta$ and $\tilde{\eta}$ we obtain the Polyakov action expressed equivalently either in open or closed variables

$$
S[X]:=-\frac{1}{2} \int d^{2} \sigma V^{T} \overline{\mathbf{G}}^{-1} V=-\frac{1}{2} \int d^{2} \sigma V^{T} \tilde{\mathbf{G}}^{-1} V .
$$

\footnotetext{
${ }^{4}$ Here, we neither need to assume that $\theta$ is Poisson nor that $F$ is closed.
} 
Actually, all this can be seen rather straightforwardly. For this, note that relations (3.7), (3.8) can alternatively be expressed as the equality of matrices $\overline{\mathbf{G}}=\tilde{\mathbf{G}}$. The relations in the form (3.10) and their transposes are obtained from the nonzero off-diagonal blocks after the similarity transformation with the block matrix $\left(\begin{array}{cc}1 & 1 \\ 1 & -1\end{array}\right)$ is applied to the equality $\overline{\mathbf{G}}=\tilde{\mathbf{G}}$.

The generalized metric $\mathbf{G}^{\prime}$ can explicitly be seen either in the Hamiltonian corresponding to the Polyakov action (4.5) or in the Hamiltonian corresponding to the action (4.2) after the equations of motions for one half of the auxiliary fields, the $\tilde{\eta} \mathrm{s}$, are used. As can straightforwardly be checked, these Hamiltonians are identical. To write down the result we introduce a new $2 n$-dimensional column vector $\Upsilon^{T}:=\left(\partial_{1} X, \eta\right)$. The auxiliary fields $\eta_{i}$ become the canonical momenta and the Hamiltonian is

$$
H[X, \eta]=\frac{1}{2} \int d \sigma^{1} \Upsilon^{T} \mathbf{G}^{\prime} \Upsilon
$$

where $\mathbf{G}^{\prime}$ the matrix given by the two equivalent decompositions (3.1) and (3.2). Hence, we have the same Hamiltonian using either the closed or the open variables. Let us note that, for $F=0$, the relation between the action (4.2) and the action (4.5) with $G^{\prime}$ expressed as in (3.1) can be found in [29]. The Hamiltonian (4.6) with $G^{\prime}$ given by (3.2) can be found, again for $F=0$, in [17]. Polyakov actions like the first one in (4.5) appeared (with $F=0$ ) in [30] in the context of Poisson-Lie T-duality.

\section{$5 \quad$ Seiberg-Witten map}

For an approach to the non-abelian case, using cohomological methods akin to the ones of Zumino's famous decent equations [31], see [32, 33]. Here we follow the approach of [3436], where it was shown that the Seiberg-Witten field redefinition from the commutative to the non-commutative setting has its origin in a change of coordinates given by a map $\rho: M \rightarrow M$, such that $\rho^{*}\left(\theta^{\prime}\right)=\theta .{ }^{5}$ This map can be derived using a generalization of Moser's lemma: Consider the family of Poisson bivectors

$$
\theta_{t}=\theta(1+t F \theta)^{-1}
$$

parameterized by $t \in[0,1]$. Of course, we have to presume that the formula is well-defined. To see that these $\theta_{t}$ are indeed Poisson for all $t$, simply observe that $G_{\theta_{t}}=e^{t F} G_{\theta}$ holds for the respective graphs. ${ }^{6}$ Partial differentiation of (5.1) with respect to $t$ leads to the differential equation

$$
\partial_{t} \theta_{t}=-\theta_{t} F \theta_{t}
$$

For $F=d A$, this can be rewritten as

$$
\partial_{t} \theta_{t}=-\mathcal{L}_{\theta_{t}(A)} \theta_{t}
$$

\footnotetext{
${ }^{5}$ As said before, here we assume only topologically trivial $[H]$-flux. The interested reader may find some relevant discussion concerning nontrivial $H$ and the related non-commutative gerbe in [37].

${ }^{6}$ Let us note again that $e^{t F} G_{\theta}$ is a bona-fide Dirac structure even for non-invertible $(1+t F \theta)$.
} 
with a vector field $\theta_{t}(A):=\theta_{t}(\cdot, A)$, with initial condition $\theta_{0}=\theta$. This differential equation can be integrated to a flow $\phi_{t}$, such that $\phi_{t}^{*}\left(\theta_{t}\right)=\theta$. Thus $\rho=\phi_{1}$. Obviously, $\rho$ explicitly depends on the choice of gauge potential $A$, hence we shall use the notation $\rho_{A}$. To avoid possible confusion, we will for a moment notationally distinguish between the tensor itself and its components in coordinates. Therefore we introduce the matrix $(\boldsymbol{\theta})^{i j}:=\theta^{i j}$. Also, denote $J_{k}^{i}=\frac{\partial \rho^{i}}{\partial x^{k}}$. We have

$$
\rho_{A}^{*}\left(\theta^{\prime k l}\right)=J_{i}^{k} J_{j}^{l} \theta^{i j}
$$

We thus get that

$$
\operatorname{det} \rho_{A}^{*}\left(\boldsymbol{\theta}^{\prime}\right)=J^{2} \operatorname{det} \boldsymbol{\theta} .
$$

Let us assume for a moment that $\boldsymbol{\theta}$ is invertible. From (3.4) we see that so is $\rho_{A}^{*} \boldsymbol{\theta}^{\prime}$. We immediately have that

$$
J^{-2}=\operatorname{det}\left(\boldsymbol{\theta}\left(\rho_{A}^{*} \boldsymbol{\theta}^{\prime}\right)^{-1}\right) .
$$

For degenerate $\theta$ and hence also $\theta^{\prime}$ the formula (5.3) still makes sense and we can argue as follows: Since the map $\rho_{A}$ is infinitesimally generated by the vector field $\theta_{t}(A)$, and the kernels of all $\theta_{t}$ 's are the same, we see that $\rho_{A}$ only changes coordinates on the symplectic leaves (of $\theta$ ). We can thus restrict ourselves to the non-degenerate case in order to carry out the computation of the Jacobian.

In the next section, we will discuss the case when the Poisson structure $\theta$ (i.e., the corresponding Dirac structure) will be used, following the suggestion of [10], to define the D-branes as its symplectic leaves. The above argument shows that we can safely restrict our discussion without the loss of generality to any of the respective D-branes. In such a case, the (Seiberg-Witten) map $\rho_{A}$ is a diffeomorphism of the D-brane world-volume $D$. The Poisson structures $\theta_{t}$ have in fact the same symplectic foliations for all $t$. Actually, all Poisson structures $\theta_{t}$, including in particular $\theta$ and $\theta^{\prime}$, are Morita equivalent [38].

Finally, on the level of Dirac structures, the Seiberg Witten map is the map of graphs $\rho^{*}: G_{\theta}^{\prime} \mapsto G_{\theta}$. More explicitly,

$$
\left\{\theta^{\prime}(\eta)+\eta, \eta \in T^{*} M\right\} \mapsto\left\{\theta(\eta)+\eta, \eta \in T^{*} M\right\}=\left\{N \theta^{\prime} N^{-T}(\eta)+N^{-T}(\eta), \eta \in T^{*} M\right\} .
$$

Hence, the Seiberg-Witten map can be seen as the map induced by the $O(n, n)$ transformation $O_{N}$ entering the decomposition (2.11), if one considers D-branes which are symplectic leaves.

\section{Noncommutative gauge theory and DBI action}

In the previous sections we have described all ingredients needed for our discussion of noncommutativity of D-branes as a consequence of their generalized geometry. Namely, we have seen that the relations (2.24), (2.25), (3.7) and the (semiclassical) Seiberg-Witten have their root in generalized geometry. Actually, it is know for quite some time [36] that the equivalence of the commutative and (semiclassically) noncommutative DBI actions follows once one has established (2.24), (2.25), (3.7) and has understood the (semiclassical) Seiberg-Witten map as a (local) D-brane diffeomorphism. Nevertheless, according to our 
best knowledge, the direct relation to generalized geometry is new. Moreover, the discussion generalizes to the case of $M$-theory branes $[24,25]$ and will be elaborated in detail in a forthcoming paper. Here we will include the derivation of the equivalence of the commutative and (semiclassically) noncommutative DBI actions for the sake of completeness and the reader's convenience. For related work based on dualities, see [39].

Assume that we have a D-brane $D$ of dimension $d$, i.e, a submanifold of target spacetime $M$ equipped with a line bundle with a connection $A$ and corresponding field strength $F$. Also, consider the restrictions (pullbacks) of the background fields (open and closed ones) to $D$. While describing the Seiberg-Witten map in the previous section, we have seen that it is quite natural to assume that there is a relation between the D-brane and the Poisson tensor $\theta{ }^{7}$ Namely, assume that our D-brane is of a particular kind, i.e., one which comes as symplectic leaf of the Poisson structure $\theta .{ }^{8}$ As argued before, under this assumption, the Seiberg-Witten map is a D-brane diffeomorphism.

Before we turn to the discussion of the DBI action and its commutative and noncommutative description, we discuss the relation between the effective closed and open string coupling constants $g_{s}$ and $G_{s}$, respectively [21]. These are related as

$$
G_{s}=g_{s}\left(\frac{\operatorname{det}(G+\Phi)}{\operatorname{det}(g+B)}\right)^{1 / 2} .
$$

We can use the formula for the determinant of a sum of a symmetric matrix $S$ and an antisymmetric matrix $A,|S+A|=|S|^{1 / 2}\left|S-A S^{-1} A\right|^{1 / 2}$, and the relation (2.24) to rewrite this as

$$
G_{s}=g_{s}\left(\frac{\operatorname{det} G}{\operatorname{det} g}\right)^{1 / 4}
$$

A most intriguing relation is obtained from (6.1) and the relation (3.7), again using the above mentioned formula for the determinant of a sum of a symmetric and an antisymmetric matrix:

$$
\frac{1}{g_{s}} \operatorname{det}^{1 / 2}(g+B+F)=\frac{1}{G_{s}} \operatorname{det}^{1 / 2}(1+\theta F) \operatorname{det}^{1 / 2}\left(G+\Phi+F^{\prime}\right) .
$$

Integrating over the D-brane world-volume

$$
\int d^{d} x \frac{1}{g_{s}} \operatorname{det}^{1 / 2}(g+B+F)=\int d^{d} x \frac{1}{G_{s}} \operatorname{det}^{1 / 2}(1+\theta F) \operatorname{det}^{1 / 2}\left(G+\Phi+F^{\prime}\right),
$$

recalling (5.3), and performing the change of coordinates according to the Seiberg-Witten map, we finally obtain a relation between the commutative and semiclassically noncommutative DBI actions

$$
S_{\mathrm{DBI}}^{c}:=\int d^{d} x \frac{1}{g_{s}} \operatorname{det}^{1 / 2}(g+B+F)=\int d^{d} x \frac{1}{\hat{G}_{s}} \operatorname{det}^{1 / 2}\left(\frac{\hat{\theta}}{\theta}\right) \operatorname{det}^{1 / 2}\left(\hat{G}+\hat{\Phi}+\hat{F}^{\prime}\right)=: S_{\mathrm{DBI}}^{\mathrm{nc}} .
$$

\footnotetext{
${ }^{7}$ Recall, in accordance with our above discussion of the open-closed relations, here we start from a given closed background $(g, B)$, pick a $\theta$ and determine uniquely the open variables $(G, \Phi)$.

${ }^{8} \mathrm{It}$ is straight-forward to modify everything to the case where the D-brane is a submanifold, such that the restriction of $\theta$ to it defines a regular Poisson structure, i.e. a Poisson structure having constant rank.
} 
The hat " " has the following meaning: On matrix elements of $\theta$ it is defined as $\hat{\theta}^{i j}:=\rho_{A}^{*}\left(\theta^{i j}\right)$, and similarly for the other objects. As a result of this definition, $\hat{F}^{\prime}$ is the semiclassically noncommutative field strength, which under the gauge transformation $\delta A=d \lambda$ transforms semiclassically noncommutatively, i.e.,

$$
\begin{aligned}
\delta \hat{F}_{i j}^{\prime} & =\left\{\hat{F}_{i j}^{\prime}, \tilde{\lambda}\right\}, \\
\tilde{\lambda} & =\left.\sum \frac{\left(\theta_{t}(A)+\partial_{t}\right)^{n}(\lambda)}{(n+1) !}\right|_{t=0} .
\end{aligned}
$$

Here, the curly bracket is the Poisson bracket corresponding to the Poisson tensor $\theta$ and $\tilde{\lambda}$ is the (semiclassical) noncommutative gauge parameter.

Let us note: The commutative DBI action $S_{\mathrm{DBI}}^{c}$ on the l.h.s. in (6.4) is the effective D-brane action obtained from the Polyakov action (4.5). Expressed directly in terms of the matrix $\tilde{\mathbf{G}}$, the action $S_{\mathrm{DBI}}^{c}$ is the integral of

$$
\operatorname{det}^{1 / 4} \tilde{\mathbf{G}}
$$

up to the inverse of the closed coupling constant $g_{s}$. Hence, an alternative - but completely equivalent - way of obtaining the relation between the commutative and semiclassically noncommutative DBI actions (6.4) is to start from the matrix equality $\tilde{\mathbf{G}}=\overline{\mathbf{G}}$. This makes the relation to the Polyakov action more transparent. We leave the details to the reader.

Finally, the Hamiltonian (4.6) can equivalently be expressed using either the "commutative" (3.1) or "noncommutative" (3.2) decompositions of the generalized metric $\mathbf{G}^{\prime}$. This is maybe the most direct hint from generalized geometry about the necessity of a relation like (6.4).

\section{Acknowledgments}

We would like to dedicate this article to Bruno Zumino on the occasion of his 90th birthday. We would like to thank Satoshi Watamura for important comments on an earlier version of the manuscript. The research of B.J. was supported by grant GAČR P201/12/G028. The research of J.V. was supported by Grant Agency of the Czech Technical University in Prague, grant No. SGS13/217/OHK4/3T/14. We also thank to DAAD (PPP) and ASCR \& MEYS (Mobility) for supporting our collaboration and gratefully acknowledge support from the DFG within the Research Training Group 1620 "Models of Gravity".

Open Access. This article is distributed under the terms of the Creative Commons Attribution License which permits any use, distribution and reproduction in any medium, provided the original author(s) and source are credited.

\section{References}

[1] N. Hitchin, Generalized Calabi-Yau manifolds, Quart. J. Math. Oxford Ser. 54 (2003) 281 [math/0209099] [INSPIRE]. 
[2] M. Gualtieri, Generalized complex geometry, math/0401221 [INSPIRE].

[3] A.S. Cattaneo, On the integration of poisson manifolds, Lie algebroids and coisotropic submanifolds, Lett. Math. Phys. 67 (2004) 33 [math/0308180].

[4] A.S. Cattaneo and G. Felder, A path integral approach to the Kontsevich quantization formula, Commun. Math. Phys. 212 (2000) 591 [math/9902090] [INSPIRE].

[5] M. Bojowald, A. Kotov and T. Strobl, Lie algebroid morphisms, Poisson $\sigma$-models and off-shell closed gauge symmetries, J. Geom. Phys. 54 (2005) 400 [math/0406445] [InSPIRE].

[6] A. Kotov and T. Strobl, Generalizing geometry - Algebroids and $\sigma$-models, arXiv: 1004.0632 [INSPIRE].

[7] A. Kotov, P. Schaller and T. Strobl, Dirac o-models, Commun. Math. Phys. 260 (2005) 455 [hep-th/0411112] [INSPIRE].

[8] C. Klimčík and T. Strobl, WZW - Poisson manifolds, J. Geom. Phys. 43 (2002) 341 [math/0104189] [INSPIRE].

[9] P. Ševera, Letters to Alan Weinstein. 2, http://sophia.dtp.fmph.uniba.sk/ severa/letters/.

[10] T. Asakawa, S. Sasa and S. Watamura, D-branes in generalized geometry and Dirac-Born-Infeld action, JHEP 10 (2012) 064 [arXiv:1206.6964] [INSPIRE].

[11] A.S. Cattaneo and G. Felder, Coisotropic submanifolds in Poisson geometry and branes in the Poisson sigma model, Lett. Math. Phys. 69 (2004) 157 [math/0309180].

[12] A. Alekseev and T. Strobl, Current algebras and differential geometry, JHEP 03 (2005) 035 [hep-th/0410183] [INSPIRE].

[13] J. Ekstrand and M. Zabzine, Courant-like brackets and loop spaces, JHEP 03 (2011) 074 [arXiv: 0903.3215] [INSPIRE].

[14] G. Bonelli and M. Zabzine, From current algebras for p-branes to topological M-theory, JHEP 09 (2005) 015 [hep-th/0507051] [INSPIRE].

[15] N. Halmagyi, Non-geometric string backgrounds and worldsheet algebras, JHEP 07 (2008) 137 [arXiv:0805.4571] [INSPIRE].

[16] D. Roytenberg, A Note on quasi Lie bialgebroids and twisted Poisson manifolds, Lett. Math. Phys. 61 (2002) 123 [math/0112152] [INSPIRE].

[17] N. Halmagyi, Non-geometric backgrounds and the first order string $\sigma$-model, arXiv:0906.2891 [INSPIRE].

[18] C.-S. Chu and P.-M. Ho, Noncommutative open string and D-brane, Nucl. Phys. B 550 (1999) 151 [hep-th/9812219] [INSPIRE].

[19] V. Schomerus, D-branes and deformation quantization, JHEP 06 (1999) 030 [hep-th/9903205] [INSPIRE].

[20] F. Ardalan, H. Arfaei and M. Sheikh-Jabbari, Noncommutative geometry from strings and branes, JHEP 02 (1999) 016 [hep-th/9810072] [INSPIRE].

[21] N. Seiberg and E. Witten, String theory and noncommutative geometry, JHEP 09 (1999) 032 [hep-th/9908142] [INSPIRE].

[22] M.R. Douglas and N.A. Nekrasov, Noncommutative field theory, Rev. Mod. Phys. 73 (2001) 977 [hep-th/0106048] [InSPIRE]. 
[23] R.J. Szabo, Quantum field theory on noncommutative spaces, Phys. Rept. 378 (2003) 207 [hep-th/0109162] [INSPIRE].

[24] B. Jurčo and P. Schupp, Nambu- $\sigma$-model and effective membrane actions, Phys. Lett. B 713 (2012) 313 [arXiv:1203. 2910] [InSPIRE].

[25] P. Schupp and B. Jurčo, Nambu $\sigma$-model and branes, PoS (CORFU2011) 045 [arXiv: 1205.2595] [INSPIRE].

[26] P. Bouwknegt, Lectures on cohomology, T-duality, and generalized geometry, Lect. Notes Phys. 807 (2010) 261.

[27] M.J. Duff and J.X. Lu, Duality rotations in membrane theory, Nucl. Phys. B 347 (1990) 394 [INSPIRE].

[28] T. Courant, Dirac manifolds, Trans. Amer. Math. Soc. 319 (1990) 631.

[29] L. Baulieu, A.S. Losev and N.A. Nekrasov, Target space symmetries in topological theories. 1, JHEP 02 (2002) 021 [hep-th/0106042] [INSPIRE].

[30] C. Klimčík and P. Ševera, Poisson-Lie T duality and loop groups of Drinfeld doubles, Phys. Lett. B 372 (1996) 65 [hep-th/9512040] [INSPIRE].

[31] B. Zumino, Cohomology of gauge groups: cocycles and Schwinger terms, Nucl. Phys. B 253 (1985) 477 [inSPIRE].

[32] D. Brace, B. Cerchiai, A. Pasqua, U. Varadarajan and B. Zumino, A cohomological approach to the nonAbelian Seiberg-Witten map, JHEP 06 (2001) 047 [hep-th/0105192] [INSPIRE].

[33] B. Cerchiai, A. Pasqua and B. Zumino, The Seiberg-Witten map for noncommutative gauge theories, talk presented at Continuous Advances in QCD 2002/Arkadyfest, May 17-23, University of Minnesota, U.S.A. (2002), hep-th/0206231 [INSPIRE].

[34] B. Jurčo and P. Schupp, Noncommutative Yang-Mills from equivalence of star products, Eur. Phys. J. C 14 (2000) 367 [hep-th/0001032] [INSPIRE].

[35] B. Jurčo, P. Schupp and J. Wess, Noncommutative gauge theory for Poisson manifolds, Nucl. Phys. B 584 (2000) 784 [hep-th/0005005] [INSPIRE].

[36] B. Jurčo, P. Schupp and J. Wess, Non-abelian noncommutative gauge theory via noncommutative extra dimensions, Nucl. Phys. B 604 (2001) 148 [hep-th/0102129] [INSPIRE].

[37] P. Aschieri, I. Bakovic, B. Jurčo and P. Schupp, Noncommutative gerbes and deformation quantization, hep-th/0206101 [INSPIRE].

[38] H. Bursztyn and O. Radko, Gauge equivalence of Dirac structures and symplectic groupoids, math/0202099.

[39] D. Brace, B. Morariu and B. Zumino, Duality invariant Born-Infeld theory, hep-th/9905218 [INSPIRE]. 